\title{
Health literacy as a challenge for health education
}

\author{
I. Stars \\ Rīga Stradinš University, Riga, Latvia
}

\begin{abstract}
Scientific literature demonstrates a well-established correlation between education and health literacy; education and health outcomes; health literacy and health outcomes. Health literacy has a mediating role between education and health. Health literacy is known as a complex concept, it has multiple definitions, underlying meanings and conceptual models. Diversity of health literacy can be a challenging motivator for health education. Indepth study of scientific literature was carried out to highlight the link between health literacy and health education and to emphasize health literacy challenges for health education. Five health literacy challenges for health education were identified: (1) health literacy as an outcome related to health education interventions; (2) multilayered health literacy or different levels of health literacy; (3) the concept of health literacy and its comprehensiveness; (4) shift from a passive to more interactive and empowering health education; (5) cultural context of health literacy and health education. Enhanced health literacy definitions and conceptual models require more complex, comprehensive, socially oriented, culture sensitive, participatory health education.
\end{abstract}

\section{Introduction}

The association between education and health is well researched, well established and strong [1]. It has received much attention and interest in scientific discourse [1] and a large body of evidence establishes a positive correlation between education, health and health outcomes [2]. The link between education and health has different potential explanations and interpretations [3], therefore this relationship is defined as a complicated one and mechanisms or pathways underlying "education - health" association have not been conclusive and accomplished [4]. Consequently, understanding the ways by which education affects individual and population health, health-related behaviour and health outcomes can be a challenging and not an easy task.

General education positively influences people's knowledge and skills (reading, writing, listening, speaking, numeracy, etc.), also promotes development of multiple literacy (for example, science, media, information, civil, cultural, etc. literacy), among them - health literacy [5]. Health literacy is defined as the cognitive and social skill that determines the motivation and ability of individuals to gain access, to understand and use information in ways that promote and maintain good health [6]. It is described as a discrete form of literacy [7], which is a context and content of specific literacy [8] that stands in its own 
right and has proved itself as a self-sufficient issue in public health, health promotion and health education discourses.

Health literacy as a distinctive concept was first used in 1974 in publication on health education as a policy issue and indicated a school health education in relation to health literacy development. Schools were seen as responsible for delivering fundamental health information on different health topics, such as safety, nutrition, physical activity, hygiene, etc. [9]. This early use of the concept of health literacy demonstrates an interconnection between health education and health literacy [10]. Currently, health literacy is conceptualized as a possible pathway by which the link between education and health can be researched, comprehended and interpreted [11]. There is evidence that low health literacy is associated with poorer health outcomes and poorer use of health care services $[12,13]$, higher medical costs [14] and lower self-reported overall health [15]. As health literacy affects health, health-related behaviour and health outcomes and comes to be a strong predictor of health, there is a need to develop, improve and promote health literacy among population. Health education interventions are critical to improve health literacy and increase health-related knowledge and competencies. Health education and health literacy are closely interconnected. Health education is directed towards improving health literacy and is presented as one of the key outcomes of health education policies, activities and interventions [16]. At the same time, health literacy is an ambiguous concept that has become a remarkable issue of today's studies [17] in health and public health sciences. Endeavour to define, redefine, conceptualize and measure health literacy leads to the condition that 'the term 'health literacy' itself has come to mean different things to various audiences and has become a source of confusion and debate" [17]. The concept and field of health literacy has evolved [18] and has expanded in its meaning [19], scope and depth [17]. Thus, multiple definitions, conceptualizations and different conceptual models of health literacy now are available [20]. It is further argued to revise the concept of health literacy and consider for a new - more comprehensive and integrative - definition of health literacy [21]. This theoretical and conceptual diversity of health literacy concept can be proposed as a challenging factor for public health and especially - for health education theory and practice.

\section{Aim and methods}

The aim of this theoretical research is to highlight a mutual relationship between health literacy and health education and identify health literacy challenges for health education. For this purpose, an in-depth scientific literature analysis was made.

\section{Results}

The literature on health literacy is gradually and constantly developing, and the quantity of scientific knowledge in theoretical aspects of health literacy is increasing. An in-depth scientific literature analysis allowed us to recognize the following health literacy challenges for health education:

\subsection{First challenge. Health literacy as an outcome related to health education interventions}

Nutbeam $[16,18]$ notes that from public health perspective health literacy can be proposed as an outcome to health education and health communication activities. Health education is focused towards developing, improving and promoting health literacy [16]. More 
specifically, health literacy can be conceptualized as a learning outcome in schools and five core components of health literacy should be taught, learned and reached - theoretical knowledge, practical knowledge, critical thinking, self-awareness, citizenship - regarding health and health-related behaviour [22].

When defining health literacy as an outcome of health education, some aspects of outcome-based education can be used in this context. An outcome - education directs every part of the educational process and educational system around the proposed and desirable outcomes - clearly state the expected knowledge, skills, attitudes and competencies. It makes the learning process more targeted and well organized because it is oriented toward educational experience and persuasion that each student should have achieved the goal [23]. Apart from this thoughtfully and clearly defined learning outcomes help to plan, organize and guide educational programs (content) and learning process (methods) [23].

Health literacy therefore offers a framework for defining learning outcomes of health education that expects to be delivered from the health education interventions and identifies intended outcomes of health education programmes. Health literacy explains the purpose of health education, also clarifies details of proposed health literacy domains. However, demands for more extensive and integrative definition of health literacy, challenge health education to be more diverse as well.

\subsection{Second challenge. Multi-layered health literacy and different levels of health literacy}

The field of health literacy has expanded and evolved noticeably over recent decades $[16,17]$. The early conceptions of health literacy focused predominantly on the functional health literacy and on an individual's ability to understand and comply with health instructions and basic health information [16]. Nowadays more than one health literacy level is outlined, for example, Nutbeam distinguishes and describes three levels or dimensions relevant to health literacy [16]:

1) basic functional health literacy is conceptualized as sufficient reading, writing, speaking, numeracy skills to be able to function effectively in everyday health contexts and situations [16];

2) communicative or interactive health literacy - more advanced cognitive and literacy skills which, together with social skills, can be used to actively participate in everyday health activities, use different forms of communication and to apply new information to changing circumstances regarding health context [16];

3) critical health literacy - the ability to critically analyse and use health information, to make informed health decisions. It is a set of skills, which enable people to participate more actively in political and social decisions affecting their health [16].

Conceptualized this way, health literacy comes to be more comprehensive and multilayered. Consequently, health education programmes and interventions should address all these dimensions of health literacy.

\subsection{Third challenge. The concept of health literacy and its comprehensiveness}

The concept of health is a multi-facetted and represents a complex system, which includes not only physical and biological well-being, but also social, mental, spiritual well-being. Health is much more than preventing ill - health (disease, disability, etc.) in individual level. It is an ambiguous and dynamic concept, which includes a mutual relationship between individuals and their social, political, cultural and economic environment [24] or 
the social determinants of health. Health education needs to recognize and consider the complexity of health and surrounding determinants of health [25].

More broad and comprehensive conceptualization of health concept determines that the definition of health literacy has been broadened and become more comprehensive as well. The field of health literacy has expanded in its scope and depth [26]. The uncertainty about the concept of health literacy can be a challenge for health education. Today health literacy goes beyond its narrow conceptualization and is much more than an individual's ability to perform basic reading, writing and numeracy tasks required for an adequate and effective functioning in the health care environment and settings [27]. New concept of health literacy means much "more than being able to read pamphlets and successfully make appointments" [16]. There is an assumption that health literacy should be considered not only as an individual issue, but also as a feature of intense and mutual reciprocity between personal health literacy, his or her health and wider social conditions and general environment [26]. Now health literacy includes more advanced and complicated skills needed to function, participate and communicate in health context, also to think critically, evaluate and use information, make informed decisions to achieve a greater control over health circumstances $[16,18]$. World Health Organization insists that health literacy should become more complex, namely, an individual behaviour-oriented communication and collaboration should be combined with the environmental, political and social factors that affect individuals and public health [25]. To avoid " 'blindness' to social aetiology of health and illness" [28], there is a need to supplement health education with more comprehensive understanding of social determinants of health. Health education, in this more comprehensive approach, should consider the complexity of health and social determinants of health - social, cultural, economic, political and environmental factors [26] influencing individual and community health context and health outcomes. The awareness of the social determinants of health can encourage and empower people and community for reasoned actions to influence and change these social, cultural, economic, political and environmental determinants of health [24, 25].

\subsection{Fourth challenge. From a passive to more interactive and empowering health education}

Dynamic and comprehensive approach to health literacy requires more interactive health education that not only gives information in a passive and directive way, but also empowers people by enhancing their ability to think of healthy behaviours and lifestyle habits, seek, critically evaluate and use health information, and also motivates people to take actions to improve their health [29] and allows them to look at health in a holistic way [24]. Such health education should address health in personal, as well - social arenas [30], as Nutbeam concludes: "Health literacy is critical to personal empowerment" [16].

Empowerment is essentially important in the context of critical health literacy, because it leads to personal and social change, it encourages people for more active and profound participation in group actions and social dialogues, it enhances control and beliefs in ability to change own lives [30]. It promotes awareness of the fact that world and life is created and lived by people [24], it contributes to a wider ability to achieve control over the determinants of health [31].

New health literacy requires more active individual participation in health education activities instead of passive receiving of health-related information diffused in a directive, top-down approach. Health education should employ methods that allow interaction, participation, critical reflections and analysis. While traditional health education programmes concentrate predominantly on providing information in a transmission approach and see learners to be simply as passive recipients, new health education has the 
advantage of enabling to engage with health information [24]. World Health Organization points out: "Such health education leads to health literacy, leading to personal and social benefit, by enabling effective community action, and by contributing to the development of social capital" [25].

\subsection{Fifth challenge. Health literacy, health education and cultural context}

Literacy, health and health literacy do not exist in a vacuum or neutral zone. It is because people develop their ideas and conceptions of literacy, health, illness and health literate person in a cultural framework that is influenced by the family and community issues, linguistic context, social and cultural factors [32]. Culture gives the meaning to health beliefs, its explanations and interpretations. Understanding of the cultural context is necessary for an in-depth understanding of health, health related-behaviour, health literacy and related meanings. Cultural and social environment and family experience affect people's beliefs about healthy lifestyle, also form the contexts in which people communicate and debate about health and health-related themes [32]. Health literacy is also affected by belief systems and communication styles of a particular community and its culture, as well as people's understanding and response styles to health-related information [32]. Health literacy as a social construct requires understanding of the local cultural and linguistic context because culture and language form the context for the acquisition and application of health literacy skills [32].

Thus, health promotion and health education interventions must consider and respond to the local cultural context [29]. Culturally and linguistically appropriate health education interventions are required. Health education must be reflective to community cultural issues regarding health and ill-health, must take into account that culture, language, and learning are closely interrelated and "to be effective, health education must be conducted in both culturally and linguistically appropriate formats to address the increasingly diverse multicultural and multilingual population" [32]. There is a need in culturally responsive health education policy and practice. Health educators must recognize this cultural and linguistic diversity of health literacy and health education: "In an environment of high diversity, the interconnections between health literacy, culture and language must also be considered in policies, practice and training" [33]. Culturally sensitive health education is respectful of the health beliefs, interpretations, practices, experience and needs of different people [32] and their worldview [33].

\section{Conclusions}

Concept of health literacy indicates to a reciprocal and meaningful association between education, literacy and health.

An impressive progress characterizes the field of health literacy. The concept of health literacy has evolved and now presents itself as a complicated and ambiguous theoretical issue that challenges traditional health education and its theoretical and methodological underpinnings.

Diversity and comprehensiveness of health literacy concept can be perceived as a challenging aspect for health education. Health literacy is not any more an individual trait or even more - deficiency. Health literacy is not simply health knowledge. Health literacy gains more wide and comprehensive definitions and conceptualizations. Multi-layered models of health literacy require more complex health education. Functional health literacy predominantly focuses on the communication of health-related information, interactive health literacy is oriented towards development of personal skills that are required in health environment, critical health literacy focuses particularly on personal and community 
empowerment [16]. Besides the functional aspects of health literacy there should be also information and awareness of the social determinants of health included into health education policies and interventions. New health education should encourage critical thinking, motivated and reasoned action and information based choices, active participation in promoting health and wellbeing.

Health literacy is an active and dynamic phenomenon, because societies and communities are changing constantly, they are not static. People from different cultural and linguistic backgrounds form different belief systems, communication styles, and understanding and response to health information.

Enhanced health literacy definitions and conceptual models require more complex, comprehensive, socially oriented, culture sensitive, participatory health education to plan more targeted health education interventions and to avoid failures in health education.

\section{References}

1. Zimmerman, E., Steven Woolf, H.S. Understanding the relationship between education and health (2014). Discussion Paper, Institute of Medicine, Washington, DC. Accessed at http://www. iom.edu/understandingtherelationship (on 16.12.2016)

2. Feinstein, L., Sabates, R., Anderson, M.T., Sorhaindo, A. and Hammond, C. What are the effects of education on health? Measuring the Effects of Education on Health and Civic

3. Engagement Proceedings of the Copenhagen Symposium (2006). Accessed at https://www. oecd.org/edu/innovation-education/37437718.pdf (on 02.12.2016)

4. Suhrcke, M., de Paz Nieves, C. The impact of health and health behaviours on educational outcomes in high income countries: a review of the evidence (2011). Copenhagen, WHO Regional Office for Europe

5. Cutler, M.D., Lleras-Muney, A. Education and Health: Evaluating Theories and Evidence. NBER Working Paper No. 12352 June, JEL No. I1, I2 (2006)

6. Education Matters for Health. Issue Brief 6: Education and Health, page 1-11(2009) Accessed at http://www.commissiononhealth.org/PDF/c270deb3ba $42-4$ fbd-baeb-2cd65956f00e/Issue $\% 20$ Brief $\% 206 \% 20$ Sept $\% 20$ $09 \% 20 \% 20$ Education $\% 20$ and $\% 20$ Health.pdf (on 02.12.2016)

7. Nutbeam, D. Health promotion glossary. Health Promotion International 13, 349364 (1998)

8. Kickbusch, S.I. Health literacy: addressing the health and education divide. Health Promotion International 16(3), 289-297 (2001)

9. Nutbeam, D. Defining, measuring and improving health literacy. HEP 42(4), 450455 (2015)

10. Simonds, S.K. Health education as social policy. Health Education Monograph 2, $1-25$ (1974)

11. Selden, C., Zorn, M., Ratzan, S.C., Parker, R.M. Health literacy. Bethesda (MD): National Library of Medicine, Feb (2000)

12. van der Heide, I., Wang, J., Droomers, M., Spreeuwenberg, P., Rademakers, J., Uiters, E. The relationship between health, education, and health literacy: results from the Dutch Adult Literacy and Life Skills Survey. Journal of Health Communication 18(Suppl. 1), 172-184 (2013)

13. DeWalt, D.A., Berkman, N.D., Sheridan, S., Lohr, K.N., Pignone, M.P., et al Literacy and health outcomes: a systematic review of the literature. Journal of General Internal Medicine 19, 1228-1239 (2004) 
14. Berkman, N.D., Sheridan, S.L., Donahue, K.E., Halpern, D.J., Crotty, K. Low health literacy and health outcomes: an updated systematic review (2011). Annals of Internal Medicine 155(2), 97-107

15. Howard, D.H., Gazmararian, J., Parker, R.M. The impact of low health literacy on the medical costs of Medicare managed care enrolees (2005). The American Journal of Medicine 118, 371-377

16. Baker, D., Parker, R., Williams, M.V., Nurss J. The Relationship of Patient Reading Ability to Self-Reported Health and Use of Health Services. American Journal of Public Health 87(6), 1027-1039 (1997)

17. Nutbeam, D. Health literacy as a public health goal: A challenge for contemporary health education and communication strategies in the 21 st century. Health Promotion International 15, 259-267 (2000)

18. Baker, D.W. The meaning and the measure of health literacy. The Journal of General Internal Medicine 21(8), 878-883 (2006)

19. Nutbeam, D. The evolving concept of health literacy. Social Science \& Medicine. 67(12), 2072-2078 (2008)

20. Zarcadoolas, C., Pleasant, A., Greer, D.S. Understanding health literacy: an expanded model. Health Promotion International 20(2), 195-203 (2005)

21. Sørensen, K., Van den Broucke, S., Fullam, J., Doyle, G., Pelikan, J., Slonska, Z., Brand, H. Health literacy and public health: a systematic review and integration of definitions and models. BMC Public Health 12, 80 (2012)

22. Pleasant, A., R.E. Rudd, C. O’Leary, M.K. Paasche-Orlow, M.P. Allen, W. Alvarado-Little, L. Myers, K. Parson, and S. Rosen. Considerations for a new definition of health literacy. Discussion Paper, National Academy of Medicine, Washington, DC (2016)

23. Pakkari, L., Paakkari, O. Health literacy as a learning outcome in schools. Health Education 112(2), 133-152 (2012)

24. Spady, W. Outcome-based Education: Critical Issues and Answers (1994)

25. Matthews, C. Critical pedagogy in health education. Health Education Journal. 73(5), 60-609 (2014)

26. WHO, Track 2: Health literacy and health behaviour. 7th Global Conference on Health Promotion: track themes. Accessed at http: //www. who. int/healthpromotion/conferences/7gchp/tra $\mathrm{ck} 2$ /en/ (on 10.01.2017)

27. Ishikawa, H., Kiuchi, T. Health literacy and health communication. Biopsychosoc Med 4, 18 (2010)

28. American Medical Association; Ad Hoc Committee on Health Literacy for the Council on Scientific Affairs. Health literacy: report of the Council on Scientific Affairs. JAMA 281, 552-557 (1999)

29. Tones, K. Radicalism and the Ideology of health education. Health Education Research 8(2), 147-150 (1993)

30. Jahan, A. Health Literacy. Promoting health literacy: a case study in the prevention of diarrhoeal disease from Bangladesh. Health Promotion International 15(4), 285 $289(2000)$

31. Wallerstein, N., Bernstein, E. Empowerment education: Freire's ideas adapted to health education (1988). Health Education Quarterly. Winter 15(4), 379-394

32. Estacio, E.V. Health literacy and community empowerment: it is more than just reading, writing and counting (2013). Journal of Health Psychology 18(8), 1056-1068

33. Institute of Medicine, Committee on Health Literacy. Health Literacy: A Prescription to End Confusion (2004) 
34. Lowell, A., Schmitt, D., Ah Chin W., Connors, C. Provider Health Literacy, cultural and communication competence: towards an integrated approach in the Northern Territory. Department of Health Library Services (2014) 